\title{
Ultra-sonografia abdominal e pélvica em cães da raça golden retriever sadios, portadores e afetados pela distrofia muscular progressiva
}

\author{
Abdominal and pelvic ultrasonography in healthy golden retriever dogs, carriers \\ and affected by gradual muscular dystrophy
}

\author{
Angélica Paula Grando ${ }^{\mathrm{I}}$ Arani Nanci Bonfim Mariana ${ }^{\mathrm{II}}$ Maria Angélica Miglino ${ }^{\mathrm{II}}$ \\ Franklin Almeida Stermann ${ }^{\mathrm{II}, \mathrm{IV}}$ Mayana Zatz ${ }^{\mathrm{III}}$ Luciane Maria Kanayama ${ }^{\mathrm{IV}}$ Matheus Levi Tjara Feitosa ${ }^{\mathrm{II}}$ \\ Daniele dos Santos Martins ${ }^{\mathrm{II}}$ Adriana Caroprezo Morini" Juliana Passos Alves dos Santos ${ }^{\mathrm{II}}$ \\ Leandro Fadel $^{\mathrm{II}}$ Flávio Ribeiro Alves ${ }^{\mathrm{II}}$ Carlos Eduardo Ambrósio ${ }^{\mathrm{I}}$
}

\section{RESUMO}

A distrofia muscular de Duchenne (DMD) é um tipo de distrofia muscular em humanos caracterizada por uma doença genética ligada ao cromossomo $X$. O cão golden retriever portador da distrofia muscular (GRMD) tem sido intensamente estudado e considerado o modelo mais representativo para a doença observada em humanos. Assim, como forma de verificar anormalidades em órgãos internos nesses animais, foi realizado o exame ultra-sonográfico de 24 cães golden retriever saudáveis, portadores e afetados pela distrofia muscular. O exame ultra-sonográfico do GRMD diagnosticou aumento hepático de moderado a severo, incluindo os vasos hepáticos e seus ramos e aumento de ecogenicidade da vesícula biliar e vesícula urinária. Entretanto, não foram observadas imagens claras de alterações no baço e nos vasos ramos da aorta. A partir disso, acreditamos que o exame ultra-sonográfico constituise em um procedimento útil no acesso de órgãos abdominais em cães afetados pela distrofia muscular.

Palavras-chave: miopatia, Duchenne, doença genética, distrofia canina.

\section{ABSTRACT}

Duchenne muscular dystrophy (DMD) is one type of human's muscular dystrophy characterized by a genetic disorder linked to the $X$ chromosome. The Golden Retriever muscular dystrophic (GRMD) has been extensively studied and considered the best resembling model to the human disease. Therefore, for identifying internal organs abnormality in GRMD, abdominal and pelvic ultrasonography was performed in 24 golden retriever dogs, either healthy or muscular dystrophic in different levels of disease. The GRMD ultrasonographic exams diagnosed moderate to severe liver enlargement, including hepatic vessels and their branches and increase of echogenicity in gallbladder and urinary bladder. However was not-clearly recognized pathologic images from spleen and aortic vessels were accessed. Therefore, we believe, the ultrasonographic exam was an useful procedure to the assessment of abdominal organs in dogs affected by muscular dystrophy.

Key words: myopathy, Duchenne, genetic disorder, canine dystrophy.

\section{INTRODUÇÃO}

As distrofias musculares progressivas (DMP's) formam um grupo de doenças humanas caracterizadas por degeneração progressiva e irreversível da musculatura esquelética (ZATZ \& PASSOS BUENO, 1995). A Distrofia Muscular de Duchenne (DMD), de origem genética e herança recessiva, tem incidência de 1 para cada 3.000 nascimentos de sexo masculino, tornando-se sintomática nos primeiros anos de vida (três a cinco anos de idade) e evoluindo para falha de deambulação

IMédica Veterinária Autônoma, Sorocaba, SP, Brasil.

"IPrograma de Pós-graduação em Anatomia dos Animais Domésticos e Silvestres, Faculdade de Medicina Veterinária e Zootecnia (FMVZ), Universidade de São Paulo (USP), Departamento de Cirurgia. Av. Prof. Dr. Orlando Marques de Paiva, 87, Cidade Universitária, 05508-270, São Paulo, SP, Brasil. E-mail: ceambrosio@usp.br. *Autor para correspondência.

IIIInstituto de Biociências (B), USP, Centro de Estudos do Genoma Humano, São Paulo, SP, Brasil.

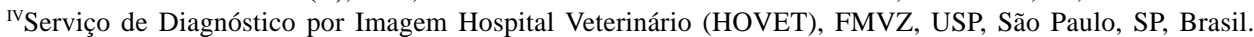


no período próximo ao final da primeira década (ENGEL, 1990; NICHOLS et al., 1994; ZATZ \& PASSOS BUENO, 1995).

BRAZEAU et al. (1992) e MORINI et al. (2008) destacam a importância do estudo de doenças humanas em modelos animais para a avaliação préclínica de possíveis modalidades de tratamento e para a deteç̧ão de anormalidades que possam alterar a farmacocinética de agentes terapêuticos administrados. No caso da DMD, o modelo animal mais estudado é o murino ( $X$-linked murine dystrophy $m d x$ ), porém, um modelo canino (canine X-linked muscular dystrophy - cxmd), devido ao seu tamanho e a sua sintomatologia semelhante à DMD vista em humanos, tem se mostrado superior (NICHOLS et al., 1994; BERGMAN et al., 2002). Embora registrada em várias raças de cães, essa distrofia tem sido mais bem caracterizada no golden retriever (Golden Retriever Muscular Dystrophy - GRMD) (BERGMAN et al., 2002).

Vários outros sistemas de órgãos musculares e não-musculares também podem estar alterados em humanos e animais distróficos. Estudos realizados por NOWAK et al. (1982); BAROHN (1988); MIYATAKE et al. (1989); MORIUCHI et al. (1991); BERRY et al. (1992); BRAZEAU et al. (1992) e STEIN et al. (2002) discorreram sobre aumentos de tensão observados na veia porta hepática, bem como flacidez da parade da vesícula biliar ocasionada pela ausência do gene da distrofina em camundongos $m d x$. Além disso, esses autores também relataram síndrome de má absorção e processos diarréicos atribuídos à disfunção pancreática em humanos.

Neste sentido, este trabalho teve como principal objetivo verificar a aparência ultra- sonográfica normal e anormal dos órgãos abdominais e pélvicos em cães da raça golden retriever, nos diversos graus de evolução da distrofia muscular.

\section{MATERIAL E MÉTODOS}

Animais

A pesquisa foi realizada após a aprovação da Comissão de Bioética da Faculdade de Medicina Veterinária e Zootecnia da Universidade de São Paulo (FMVZ-USP). Foram utilizados 24 cães da raça golden retriever entre machos e fêmeas, filhotes e adultos, portadores e afetados pela distrofia muscular progressiva. Os animais eram provenientes do canil experimental Golden Retriever Muscular Dystrophy (GRMD - Brasil) do Departamento de Cirurgia (Figura 1A). Dos 24 animais avaliados, 15 eram filhotes com seis meses de idade e nove eram adultos entre dois e cinco anos de idade. Entre os filhotes, quatro eram sadios (duas fêmeas e dois machos), cinco portadores (fêmeas) e seis afetados (machos). Entre os adultos, quatro eram sadios (duas fêmeas e dois machos), quatro portadores (fêmeas) e um afetado (macho) (Figura 1B). Os animais eram mantidos sob cuidados médicos veterinários, alimentação, higiene e recreação permanente.

\section{Diagnóstico genético}

A identificação genotípica da distrofia muscular foi realizada pelo Centro de Estudos do Genoma Humano da Universidade de São Paulo (IB-USP), logo após os nascimentos, a partir de sangue do cordão umbilical, por meio da extração genômica de DNA, usando o kit GFX Genomic Blood DNA Purification Kit (GE Healthcare). A confirmação da distrofia muscular

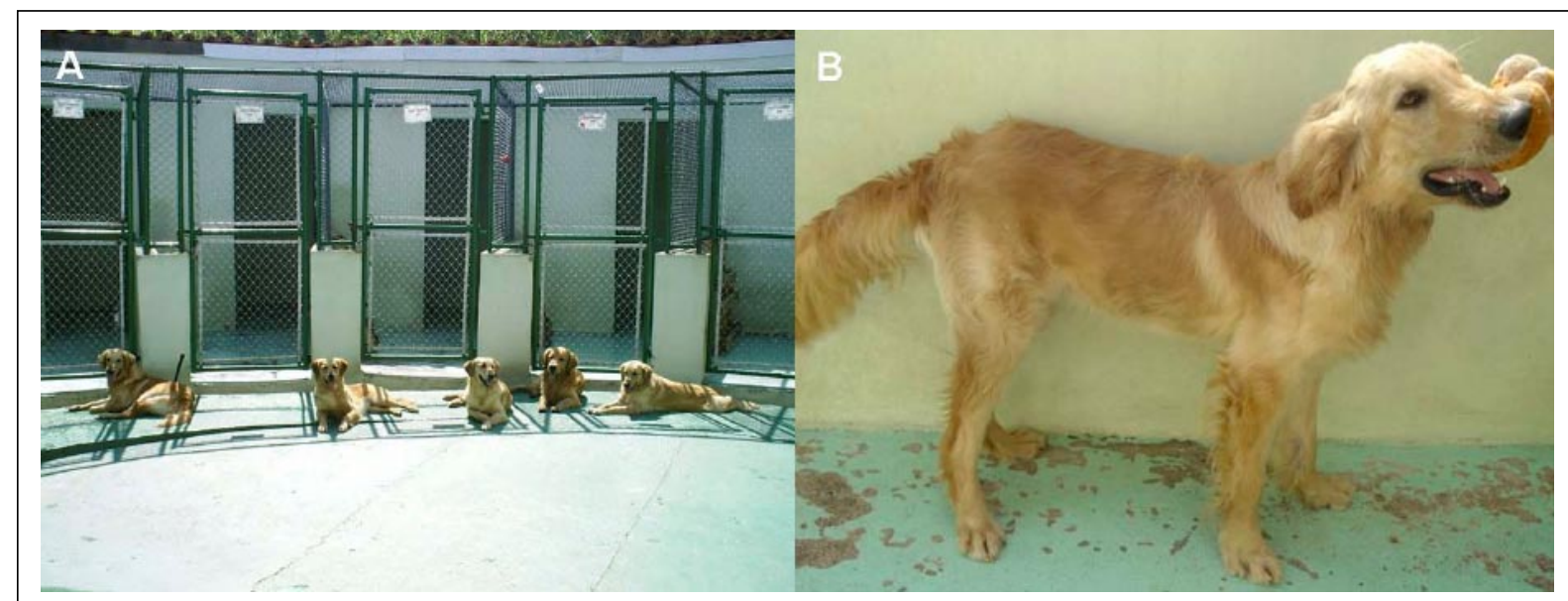

Figura 1 - A. Instalações do canil Golden Retriever Muscular Dystrophy (GRMD). B. Cão GRMD adulto, pertencente ao canil GRMD. 
também foi realizada por meio de os níveis elevados da enzima sérica creatina kinase, Kerkis et al. (2008).

Exame ultra-sonográfico

Procedeu-se o estudo ultra-sonográfico por meio de varredura abdominal e pélvica para visualização e caracterização de suas estruturas. Para isso, utilizou-se um aparelho de ultra-som portátil Tokimec ${ }^{\circledR}$, modelo CS-3030, com transdutores lineares de 7,5MHz e convexo de 5,0MHz. As imagens foram registradas em impressora video copy processor, modelo P66E, da marca Mitsubishi ${ }^{\circledR}$. Como meio de contato para transmissão de ultra-sons, foi utilizado o Carbogel $^{\circledR}$.

Para o exame ultra-sonográfico, os cães foram submetidos a jejum de sólidos por 12 horas e hídrico por pelo menos 1 hora antes do exame. Após tricotomia da região abdominal e pélvica, seguiu-se o exame, estabelecido ao acaso, sem conhecimento do executor dos exames, de qual animal era normal ou afetado, perfazendo um estudo cego do grupo. Os órgãos abdominais e pélvicos foram analisados quanto a sua topografia e aparência ultra-sonográfica.

\section{RESULTADOS E DISCUSSÃO}

Estudos realizados por NOWAK et al.(1982), BAROHN et al.(1988), MIYATAKE et al.(1989), MORIUCHI et al.(1991), BERRY et al.(1992), BRAZEAU et al.(1992) e STEIN et al.(2002) demonstraram alterações em órgãos internos musculares e parenquimatosos, tanto em indivíduos com Duchenne, como em animais com distrofia muscular. Resultados semelhantes obtidos neste experimento estão descritos na tabela 1 . De igual modo, neste trabalho foram observadas alterações marcantes da ecotextura e padrões de tamanho, dimensões e volume das estruturas abdominais e pélvicas. Recentemente, BRUMITT et al. (2006) descreveram para cães da mesma raça apenas uma hepatomegalia associada a discretas modificações da serosa abdominal, ao contrário das modificações detalhadas das estruturas abdominais aqui mostradas e enfatizadas (Tabela 1).

Apesar de a conhecida hepatomegalia neonatal descrita por ROTHUIZEN (2001), esta alteração foi observada em todos os cães distróficos do grupo estudado, corroborando as informações descritas em humanos com Duchenne (STEIN et al., 2002) na distrofia muscular hipertrófica em felinos (BERRY et al., 1992) e em camundongos $m d x$ (BRAZEAU et al., 1992). Além disso, a hepatomegalia também foi observada em outros seis cães filhotes
Tabela 1 - Porcentagens dos achados ultra-sonográficos em fígado, vesícula biliar, bexiga urinária, glândula adrenal, pâncreas e baço nos animais sadios, afetados e portadores de distrofia muscular do labrador retriever (GRMD)

\begin{tabular}{|c|c|c|c|}
\hline $\begin{array}{l}\text { Achados } \\
\text { ultra-sonográficos }\end{array}$ & Cães sadios & $\begin{array}{c}\text { Cães } \\
\text { afetados }\end{array}$ & $\begin{array}{c}\text { Cães } \\
\text { portadores }\end{array}$ \\
\hline \multicolumn{4}{|l|}{ Fígado } \\
\hline Hepatomegalia & $75 \%$ & $100 \%$ & $44,4 \%$ \\
\hline Textura hiperecóica & $87,5 \%$ & $83,3 \%$ & $88,8 \%$ \\
\hline \multicolumn{4}{|l|}{ Vesícula biliar } \\
\hline Lama biliar & $0 \%$ & $50 \%$ & $0 \%$ \\
\hline Colelitíase & $0 \%$ & $16,6 \%$ & $0 \%$ \\
\hline Pontos hiperecóicos & $0 \%$ & $0 \%$ & $11,1 \%$ \\
\hline \multicolumn{4}{|l|}{ Vesícula urinária } \\
\hline Pontos hiperecóicos & $0 \%$ & $50 \%$ & $0 \%$ \\
\hline \multicolumn{4}{|l|}{ Glândula adrenal } \\
\hline Aumento de volume & $0 \%$ & $16,6 \%$ & $0 \%$ \\
\hline \multicolumn{4}{|l|}{ Pâncreas } \\
\hline Visualização & $\mathrm{NV}^{\mathrm{a}}$ & $83,3 \%$ & $\mathrm{NV}^{\mathrm{a}}$ \\
\hline \multicolumn{4}{|l|}{ Baço } \\
\hline $\begin{array}{l}\text { Pontos hiperecóicos } \\
\text { no parêquima }\end{array}$ & $0 \%$ & $16,6 \%$ & $0 \%$ \\
\hline
\end{tabular}

(sadios e portadores) e em três adultos. Desde modo, confirmamos esses achados em cães GRMD adultos, tal qual descreveu BRUMITT et al. (2006). Somam-se ainda os achados microscópicos descritos por SANTOS et al. (2007), que descreveram a perda de organização trabecular e cordonal dos hepatócitos, a presença de células mononucleares em sinusoides e vasos, a hiperplasia dos espaços-porta e o tecido fibroso evidente, bem como áreas de congestão e isquemia em cães acometidos pela distrofia muscular.

A diminuição da ecogenicidade hepática observada por BERRY et al. (1992) em gatos distróficos não está de acordo com os achados de hiperecogenicidade hepática nos cães jovens e distróficos desta pesquisa, quando observamos que nove cães filhotes, entre sadios e portadores, e seis adultos também apresentaram hiperecogenicidade hepática. Apesar de muitas vezes tais achados ultrasonográficos constituírem quadros de infiltração gordurosa, hepatopatia por esteróides, diabetes mellitus, linfoma e hepatopatias tóxicas (MAMPRIM, 2004), atribuímos a alteração ao excesso de metabólitos oriundos de compostos degradados da musculatura esquelética e ao fato desses animais apresentarem ALT elevada, com uma resposta ao efeito da distrofia muscular (MORINI, 2008). 
O grande calibre dos ramos da veia hepática constitui-se um achado anormal, quando comparados a valores observados na literatura e descritos por SALGADO et al. (2007). Com base nas observações feitas por CHETBOUL et al. (2004) e PELLEGRINO et al. (2007) quando estudaram, respectivamente, cães distróficos e sadios, acreditamos em uma doença cardíaca concomitante como possível causa para a alteração observada ultra-sonograficamente, corroborando ainda as observações feitas por MAMPRIM (2004) em estudo semelhante. Do mesmo modo, SAMIEL (2000) relata a idade avançada como um agente colaborador no desenvolvimento de cardiomiopatias e congestão do sistema venoso hepático em cães distróficos.

A presença de sedimento biliar permanece por ser estabelecido, muito embora seja comum observá-lo em animais idosos, obesos, sedentários ou endocrinopatas (CENTER, 1992; MAMPRIM, 2004). Os cães avaliados apresentavam acentuada disfagia devido à ingestão de pequenas quantidades de alimento, justificando-se assim a ocorrência da estase biliar e a conseqüente formação de bile densa, bem como a presença de cálculos biliares em quatro desses animais (Figura 2). Tais cálculos biliares também podem estar associados a alterações nos níveis de colesterol e de componentes iônicos da bile, como foi descrito por MAMPRIM (2004). As deficiências de contração da musculatura lisa da vesícula biliar observadas neste estudo são sustentadas nas observações de NOWAK et al. (1982), BAROHN et al. (1988) e MIYATAKE et al. (1989), quando sugeriram a disfunção sistêmica da musculatura lisa vascular de camundongos $m d x$ e da motilidade gástrica em humanos com Duchenne.

Embora a esplenomegalia estivesse presente nos indivíduos distróficos estudados por STEIN et al. (2002) e BERRY et al. (1992), neste estudo, não foi observado aumento das dimensões esplênicas nos cães afetados. Em seus estudos, TANNOUZ (2004) ressaltou o volume esplênico diminuído em casos de caquexia e desidratação, condições observadas com frequência nos cães distróficos. Tais achados são também observados nos trabalhos de MORIUCHI et al. (1991), que verificaram perda de peso

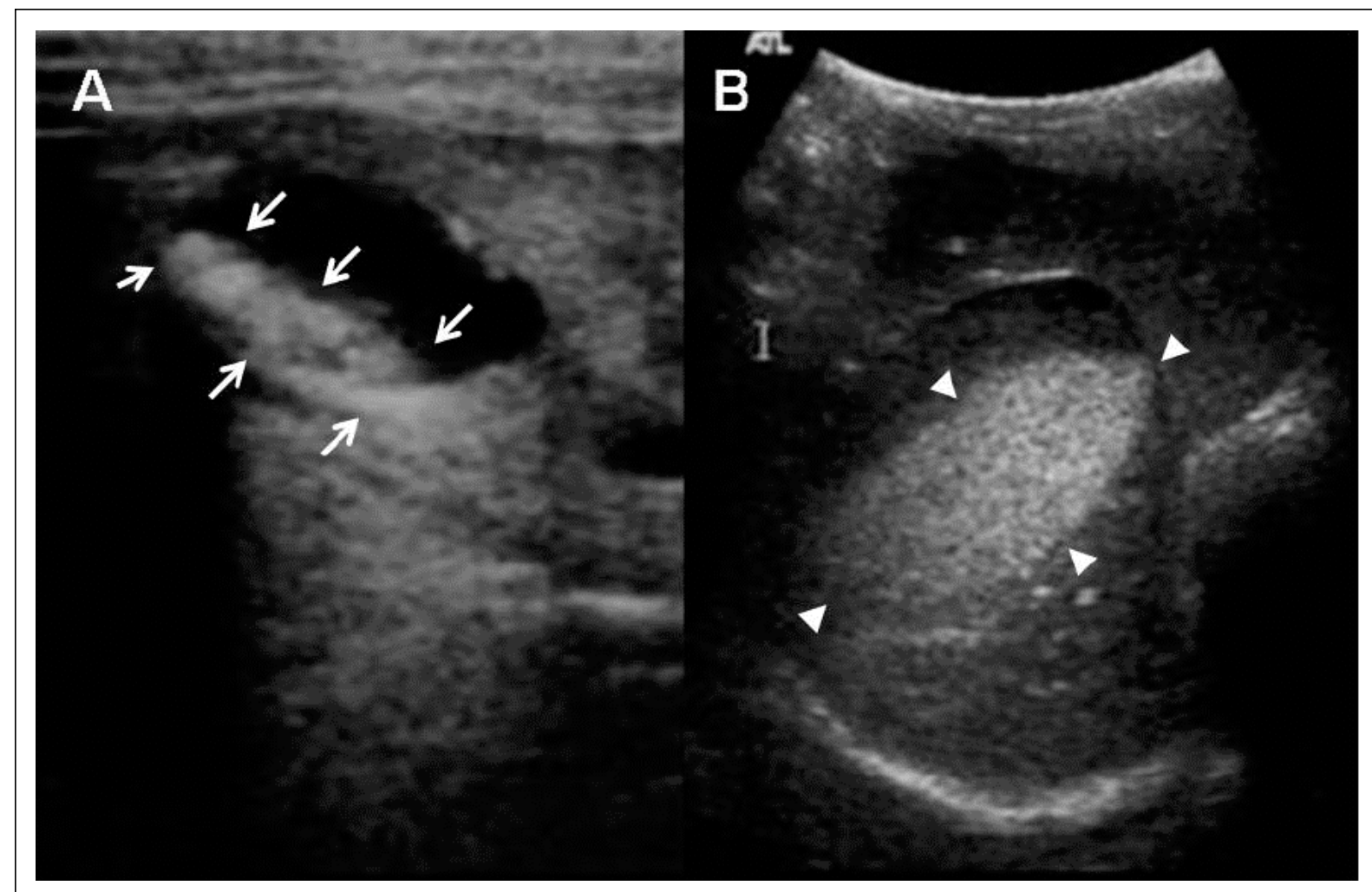

Figura 2 - A. Imagem ultra-sonográfica da vesícula biliar com presença de cálculos em seu interior em cão GRMD de 6 meses de idade. B. Imagem ultra-sonográfica da vesícula biliar com presença de bile densa em cão GRMD adulto. 
corpóreo em pacientes humanos acometidos pela DMD devido à redução da musculatura esquelética. Além disso, os cães distróficos estudados eram menores em termos de tamanho e peso corpóreo em relação aos cães sadios da mesma raça e idade, sendo esta característica cada vez mais evidente com a progressão da doença.

Na avaliação ultra-sonográfica, três cães distróficos apresentaram pontos hiperecóicos em suspensão no conteúdo da vesícula urinária. Estudos realizados por MACLEO et al. (2003) e TUBBS \& OAKES (2004) em humanos com DMD também verificaram alterações do trato urinário inferior, como, por exemplo, pequena capacidade de retenção, hiperreflexia e discinergia da musculatura do esfíncter.

Embora MUSCATELLI etal.(1994)ePILLERS et al. (1990) relatem a possibilidade da hipoplasia congênita da glândula adrenal em pacientes com distrofia muscular de Duchenne e BERRY et al. (1992) e CARPENTER et al. (1989) tenham observado a presença de mineralização das glândulas adrenais em dois felinos distróficos, neste estudo, tal alteração foi detectada apenas de forma isolada (glândula adrenal direita medindo 4,1 x 2,1cm) em um cão afetado adulto.

O pâncreas foi visibilizado em cinco dos 24 cães GRMD estudados, identificando-se a veia pancreaticoduodenal e o ducto pancreático (Figura 3A e B). O padrão corporal de menor peso e pouca gordura nos animais estudados pode ter favorecido a identificação do pâncreas, corroborando as avaliações de BERFORD (2004), o qual constatou que, para animais magros, as margens pancreáticas são discretamente mais definidas. Em um filhote afetado, também foi observada ecogenicidade pancreática elevada, contudo, consistindo em apenas um achado ocasional.

\section{CONCLUSÕES}

O exame ultra-sonográfico constituiu-se em uma técnica não-invasiva, rápida, de fácil acesso e sensível na identificação de modificações de ecogenicidade parenquimal de órgãos abdominais e pélvicos, em cães afetados pela distrofia muscular. $\mathrm{O}$ aumento de ecogenicidade e as alterações do padrão de arquitetura de órgãos como o fígado e o pâncreas, observados por meio de exame ultra-sonográfico, são sinais eficientes para realizar previsões sobre a evolução da distrofia muscular em cães, constituindose em um modelo animal adequado para estudo de alterações semelhantes na espécie humana.

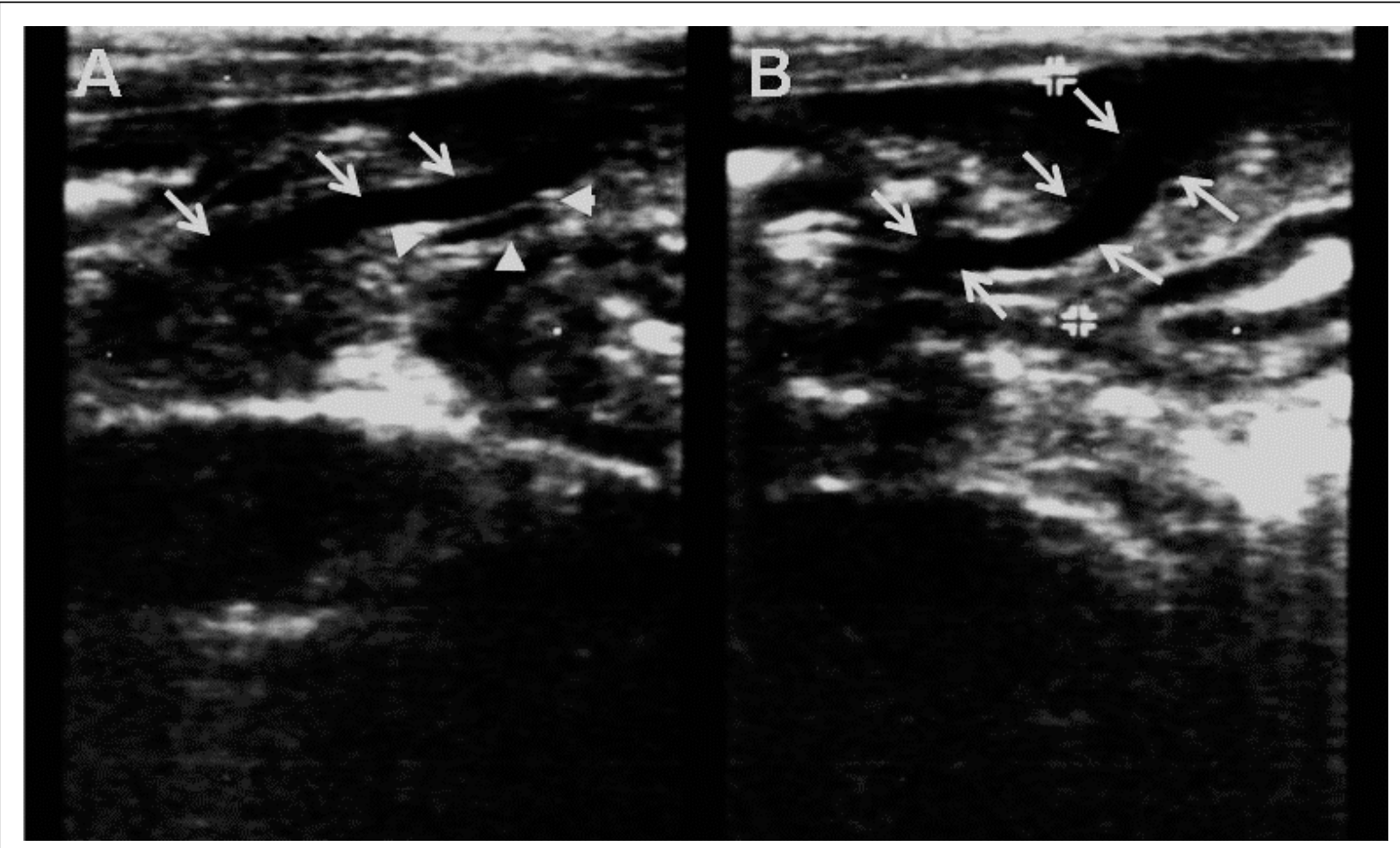

Figura 3 - A. Imagem ultra-sonográfica da veia pancreaticoduodenal (setas) e ducto pancreático (cabeças de seta) em cão GRMD de 6 meses de idade. B. Imagem ultra-sonográfica da veia pancreaticoduodenal em cão GRMD de 6 meses de idade (setas). 


\section{REFERÊNCIAS}

BAROHN, R.J. et al. Gastric hypomotility in Duchenne's muscular dystrophy. New England Journal of Medicine, v.319, p.15-18, 1988.

BERFORD, R.M. Pâncreas. In: CARVALHO, C.F. (Ed.). Ultra-sonografia em pequenos animais. São Paulo: Roca, 2004. p.75-83.

BERGMAN, R.L. et al. Dystrophin-deficient muscular dystrophy in a Labrador Retriever. Journal of American Animal Hospital Associassion, v.38, n.3, p.255-261, 2002.

BERRY, C.R. et al. Radiographic and ultrasonographic features of hypertrophic feline muscular dystrophy in two cats. Veterinary Radiology and Ultrasound, v.33, n.6, p.357364,1992

BRAZEAU, G.A. et al. Serum and organ indices of the mdx dystrophic mouse. Research Communications in Chemical Pathology and Pharmacology, v.77, n.2, p.179189, 1992.

BRUMITT, J.W. et al. Radiografic features of golden retriever muscular dystrophy. Veterinary Radiology \& Ultrasound, v.47, n.6, p.574-580, 2006.

CARPENTER, J.L. et al. Feline muscular dystrophy with dystrophin deficiency. American Journal Pathology, v.135, p.909-919, 1989

CENTER, S.A. Fisiopatologia e diagnóstico laboratorial das moléstias hepáticas. In: ETTINGER S.J. (Ed.). Tratado de medicina interna veterinária: moléstias do cão e do gato. São Paulo: Manole, 1992. p.1487-1546.

CHETBOUL, V. et al. Tissue Doppler imaging detects early asymptomatic myocardial abnormalities in a dog model of Duchenne's cardiomyopathy. Preclinical Research, v.25, p.1934-1939, 2004b

ENGEL, A.G. Doenças musculares (miopatias) e da junção neuromuscular. In: WYNGAARDEN J.B.; SMITH Jr L.H. (Ed.). Cecil - tratado de medicina interna. Rio de Janeiro: Guanabara Koogan, 1990. p.1988-2001.

KERKIS, I. et al. Early transplantation of human immature dental pulp stem cells from baby teeth to golden retriever muscular dystrophy (GRMD) dogs: local or systemic? Journal of Translational Medicine, v.6, p.35, 2008

MAMPRIM, M.J. Fígado e vesícula biliar. In: CARVALHO C.F. (Ed.). Ultra-sonografia em pequenos animais. São Paulo: Roca, 2004. p.51-73.

MIYATAKE, M. et al. Possible systemic smooth muscle layer dysfunction due to a deficiency of dystrophin in Duchenne muscular dystrophy. Journal of the Neurological Sciences, v. 93, p.11-17, 1989.

MORINI et al. Análise das dosagens e concentrações séricas da ciclosporina A em cães da Raça Golden Retriever normais ou afetados pela distrofia muscular. Brazilian Journal of Veterinary Research and Animal Science. v.45, n.2. p.131-137, 2008.
MORIUCHI, T. et al. Autopsy study on the heart, liver, kidney and brain in Duchenne muscular distrophy. Tokushima Journal of Experimental Medicine, v.38, p.5-13, 1991.

MUSCATELLI, F. et al. Mutations in the DAX-1 gene give rise to both $\mathrm{X}$-linked adrenal hypoplasia congenita and hypogonadotropic hypogonadism. Nature, v.372, n.6507, p.672-676, 1994.

NICHOLS, P.L. et al. Gene therapy in duchenne muscular dystrophy. Nih Guide, v.23, n.7, 1994. Acesso em 11 jun 2005. Online. Disponível em: <http:/grants2.nih.gov/grants/ guide/pa-files/PA-94-040.html>.

NOWAK, T.V. et al. Gastrointestinal manifestations of the muscular dystrophies. Gastroenterology, v.82, p.800-810, 1982

PELLEGRINO, A. et al. Padronização de parâmetros ecocardiográficos de cães Golden Retriever clinicamente sadio. Ciência Rural, v.37, n.4, p.1039-1044, 2007.

PILLERS, D.A. et al. Aland Island eye disease (ForsiusEriksson ocular albinism) and an Xp21 deletion in a patient with Duchenne muscular dystrophy, glycerol kinase deficiency, and congenital adrenal hypoplasia. American Journal of Medical Genetics, v.36, n.1, p.23-28. 1990.

ROTHUIZEN, J. Hepatopatias e doenças do trato biliar. In: DUNN J.K. (Ed.). Tratado de medicina de pequenos animais. São Paulo: Roca, 2001. p.444-493.

SALGADO, S.A.B. et al. Identificação das regiões correspondentes aos lobos hepáticos de cães por meio da ultrasonografia. Ciência Animal Brasileira, v.8, n.3, p.545558, 2007.

SAMIEL, H.V. Genetic surgery for muscular dystrophy in golden retrievers. Genome News Network, June, 2000. Acesso em 2003. Online. Disponível em: http:// www.genomenewsnetwork.org/articles/06_00/ muscular_dystrophy.shtml.

SANTOS, F.M. et al. Estudo microscópico do fígado e pâncreas de cães golden retriever acometidos por distrofia muscular. International Journal of Morphology, v.25, n.1, p.19293, 2007.

STEIN, M.T. et al. Fatigue, decrease interest in play, motor delay, and elevated liver function tests in a 4-year-old boy. Journal of Developmental and Behavioral Pediatrics, v.23, n.1, p.37-41, 2002.

TANNOUZ, V.G.S. Baço. In: CARVALHO C.F. (Ed.). Ultrasonografia em pequenos animais. São Paulo: Roca, 2004. p.85-99.

TUBBS, R.S.; OAKES W.J. Urinary incontinence in a patient with Duchenne muscular dystrophy and cord in the normal position with fatty filum terminale. Child's Nervous System, v.20, p.717-719, 2004.

ZATZ, M.; PASSOS BUENO, M.R. Miopatias hereditárias: avanços dos últimos dois anos. In: NITRINI R. et al. Condutas em neurologia. São Paulo: Clínica Neurológica HC-FM, USP, 1995. p.71-78. 